\title{
Cooperative Fair Scheduling for the Downlink of CDMA Cellular Networks
}

\author{
Mehrdad Dianati, Student Member, IEEE, Xuemin (Sherman) Shen, Senior Member, IEEE, and \\ Kshirasagar Naik, Member, IEEE
}

\begin{abstract}
In this paper, we study cooperation among the adjacent base stations (BSs) for downlink scheduling in code division multiple access cellular networks. We propose a cooperative fair scheduling scheme, namely, cooperative utility fair scheduling, to increase multiuser diversity gain and reduce interference among BSs. The scheduler maintains fairness and smooth service delivery by balancing the long-term average throughput of users. Monte Carlo simulation results are given to demonstrate the effectiveness of the proposed scheme in terms of multiuser diversity gain, throughput, and fairness.
\end{abstract}

Index Terms-Code division multiple access (CDMA) cellular networks, cooperative communications, fair scheduling, Monte Carlo simulations.

\section{INTRODUCTION}

$\mathbf{E}$ FFECTIVE resource management can provision quality of service (QoS) for network users and increase revenue for network operators. A typical resource management policy consists of three main elements: 1) access control to regulate the incoming traffic; 2) admission control to avoid system overload; and 3) dynamic resource allocation to efficiently share the system resources among admitted users. Scheduling is an effective technique for dynamic resource allocation in delay tolerant systems in order to achieve fairness and efficient utilization of network resources. Designing efficient scheduling schemes has been extensively investigated for wired networks [1]. However, due to the significantly different characteristics of wireless channels, the scheduling schemes perform poorly in wireless networks [2]. Since the quality of a wireless channel is a random process, and its statistical characteristics depend on the location of a mobile user, efficient utilization of bandwidth while maintaining fairness among users is a very challenging problem.

Manuscript received August 16, 2005; revised April 3, 2006 and July 9, 2006. This work was supported by a research grant from Bell University Laboratories under the sponsorship of Bell Canada and the Natural Sciences and Engineering Research Council of Canada. The review of this paper was coordinated by Prof. T. Hou.

The authors are with the Centre for Wireless Communications, Department of Electrical and Computer Engineering, University of Waterloo, Waterloo, ON N2L 3G1, Canada (e-mails: mdianati@bbcr.uwaterloo.ca; xshen@bbcr. uwaterloo.ca; knaik@bbcr.uwaterloo.ca).

Color versions of one or more of the figures in this paper are available online at http://ieeexplore.ieee.org.

Digital Object Identifier 10.1109/TVT.2007.897209
It has been demonstrated that diversity techniques provide effective solutions to mitigate the negative impacts of random channel fluctuations in wireless networks [3]. In cellular networks, the existence of multiple base stations (BSs) naturally provides a dimension of space diversity, which is referred to as the BS diversity throughout this paper. BS diversity has been applied to soft handoff in IS-95 code division multiple access (CDMA) cellular networks to increase the reliability of communications for the users near cell boundaries, where there are many simultaneous voice transmissions. For nonreal-time data traffic, simultaneous transmissions are not necessary. If certain delay can be tolerated, transmissions can be scheduled. An efficient scheduling technique is to prioritize transmission to different users based on quality of their channels. This can be considered as an opportunistic service discipline. Opportunistic scheduling can be combined with BS diversity in a selection manner to improve bandwidth utilization. If the partial channel state information (CSI) from several BSs to a group of users is available in every scheduling epoch (i.e., time slot), a scheduler can coordinate transmissions from those BSs. Furthermore, transmission power of the coordinated BSs can be optimized to improve system throughput. This can be considered as a cooperative scheduling scheme, which increases system throughput by 1) reducing interference among the adjacent BSs and 2) improving multiuser diversity gain.

In this paper, we consider scheduling for the downlink of CDMA cellular network. Downlink is the primary focus, since data traffic is expected to be heavier for the downlink than the uplink. Thus, efficient scheduling for the downlink can significantly improve the revenue of a network operator. We propose a cooperative utility fair scheduling (CUFS) scheme, where the coverage area of the cellular network is divided into many cells, and each cell is covered by a group of three most interfering sector antennas (SAs) from three adjacent BSs. There is one scheduler in each group. The scheduler implements a utility-fair [4] selection mechanism in order to select three users from three adjacent cells for transmission in each time slot. The user selection mechanism maintains the long term fairness among users. In addition, the mechanism defines a parameter to balance long term fairness and service smoothness allowing a network operator to utilize its bandwidth efficiently. An optimal power allocation scheme is introduced to minimize interference among three coordinated SAs, thereby maximizing the sum of instantaneous transmission rates from a group of adjacent SAs, which is called a cooperation group. Monte Carlo simulation results are provided to demonstrate the efficacy 
of the CUFS scheme. The contributions of this paper are threefold.

1) A utility-fair user selection mechanism is proposed to maintain long-term fairness among users and provide a flexible mechanism to control smoothness of service according to the QoS requirements of users.

2) An optimal power allocation scheme is proposed to minimize interference among coordinated SAs belonging to a cooperation group.

3) It is demonstrated that multiuser diversity gain can be improved by cooperative scheduling.

The rest of this paper is organized as follows. In Section II, we discuss related works. We specify our system model in Section III. In Section IV, we propose the cooperative fair scheduling scheme. Simulation results are given in Section V, followed by the conclusions in Section VI, respectively.

\section{RELATED WORKS}

In general, wireless scheduling schemes can be divided into two categories: 1) scheduling schemes for fixed transmission rate BSs and 2) scheduling schemes for dynamic transmissionrate BSs. For the fixed transmission rate, it is assumed that there is a power control mechanism such that a BS can transmit to all users with a fixed transmission rate. Since power control cannot compensate for channel attenuation during deep fading, a channel monitoring mechanism is usually employed by the scheduler to account for bad channel conditions. If the channel quality for a particular user is below a certain threshold, the scheduler defers transmitting to the user until its channel recovers back to a normal condition. Due to location dependence of wireless channels, some users may experience a bad channel condition more often than other users. Thus, to maintain some degree of fairness among users, a compensation mechanism is usually implemented. Some well-known scheduling schemes for the fixed transmission rate BSs are the following: 1) channel state dependent packet scheduling [5]; 2) idealized wireless fair queuing; and 3) Channel-condition Independent Fair Queuing. A survey of this type of scheduling schemes can be found in [2].

In dynamic transmission-rate systems, BS always transmits at its maximum allowable power. However, the transmission rate is adapted according to the instantaneous quality of channels for different users. In fact, transmitting at a fixed rate for all users is neither possible nor optimal. An optimal scheduling strategy to maximize the total system throughput is to transmit to only one user with the best channel quality at the maximum achievable rate in each time slot [6], [7]. This scheduling strategy can be considered as an opportunistic service discipline which has been used in several existing scheduling schemes such as the proportional fair scheduling (PFS) [4]. Although opportunistic service discipline maximizes the total bandwidth utilization, it is extremely unfair when the temporal and probabilistic characteristics of the channels from a BS to different MSs are not identical. Users with a relatively better average channel quality enjoy higher average transmission rates than those with a relatively poor average channel quality. Therefore, a fairness enforcement mechanism is necessary.
For a scheduler with an opportunistic service discipline, the higher is the number of users, the higher is the system throughput. This property is know as multiuser diversity gain [9]. The achievable gain of an opportunistic scheduler is higher when the dynamic range of channel fluctuations is larger. A multiple antenna transmission scheme, namely opportunistic beam-forming, is proposed in [9] to enrich the dynamic range of channel fluctuations. The scheme is to split the transmission power among multiple transmit antennas, where each antenna is assigned a random proportion of the total transmission power. The proportion of the power and the transmitted signal phase for each antenna can be dynamically adjusted to induce constructive and destructive addition of signals from different antennas at the receiver. This scheme can improve multiuser diversity gain if the users are fixed or if the mobility of users is very low. However, it is less effective for the independent Rayleigh fading environment, where transmit antennas are sufficiently apart. Furthermore, the rates of artificially induced random variations of signal power and phase from different antennas have to be fast enough to induce appropriate artificial fading. On the other hand, the random variations need to be slow enough to ensure the accuracy of channel estimation for proper operation of the scheduler. Our proposed scheme can enhance the channel fluctuations to increase multiuser diversity gain. However, unlike [9], we do not use artificially induced fluctuations. Instead, we employ BS selection diversity to enrich channel fluctuations and increase the effective number of users for the single scheduler by cooperative scheduling.

Opportunistic scheduling for multiple-input and multipleoutput (MIMO) systems has been studied in [10]. It is concluded that in MIMO systems, the multiuser diversity gain declines as the number of antennas increases in the absence of the partial CSI at transmitter side. As we will demonstrate in this paper, selection diversity using partial CSI increases multiuser diversity gain. Resource allocation and scheduling in multiple antenna systems have also been studied in [11] and [12]. However, the issue of fair scheduling is not properly addressed.

\section{SySTEM MODEL}

We consider the downlink of a CDMA cellular network, connected to the Internet backbone through a gateway, as shown in Fig. 1. The incoming data from the Internet backbone is routed through the gateway to the corresponding $\mathrm{BS}$ controller (BSC)/radio network controller (RNC), depending on the location of the end users. Each BSC controls multiple BSs and each BS has three SAs. The SAs are directional antennas, as shown in Fig. 2, roughly covering a $120^{\circ}$ sector of a single cell. A cooperation group consists of three SAs associated with a single scheduler, and the three SAs belong to three adjacent BSs. Each scheduler is part of a resource management module which resides in the BSC/RNC, as shown in Fig. 3. Besides the scheduling unit, the resource management module includes additional units: 1) an access control unit to regulate the incoming traffic; 2) a call admission control unit to manage call level activities; and 3) data buffers to separately 


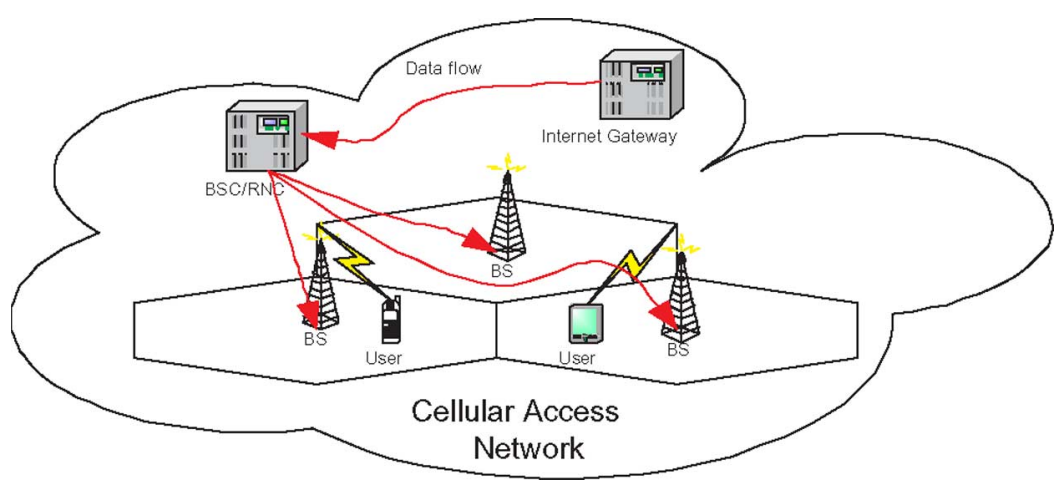

Fig. 1. Network model.

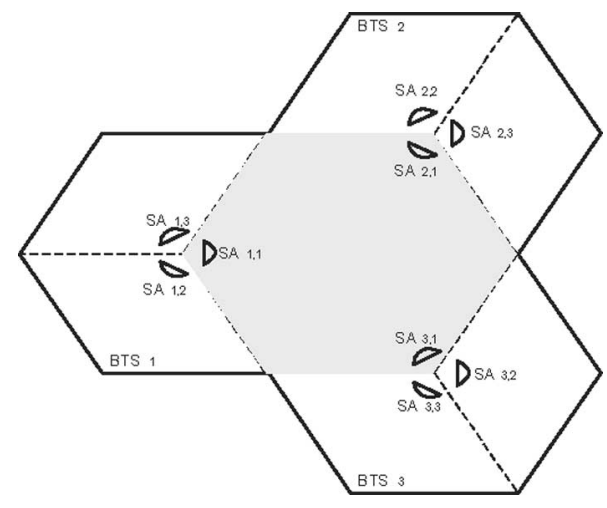

Fig. 2. Sector antennas.

buffer the incoming data for the admitted users. The resource management unit interacts with the radio unit, which is the air interface of a cellular system. It is a two-way interaction to transmit data and acquire the partial CSI of the admitted users. The scheduling unit comprises subunits for channel monitoring, QoS provisioning, queue monitoring, and transmission scheduling. The channel monitoring unit communicates with the radio unit to collect and store feedback information from users, including the estimation of channel quality from three different SAs associated with a single scheduler. The queue monitoring subsystem overlooks the queue accumulations to adjust users service rates if necessary. The QoS provisioning unit interacts with the call admission unit to allocate proper share of the wireless channel bandwidth to users according to requirements the users and the service contracts.

The air interface can support several key features as follows. Adaptive Modulation and Coding (AMC) with higher order modulation of QPSK, 8PSK, and 16 QAM enables multirate transmission. The BS has a basic scheduling granularity of 1.25 -ms time slots. There is a forward packet data control channel to carry user's MAC ID, packet size, and other necessary information for proper reception of the transmitted data packets. A reverse channel quality indicator channel (R-CQICH) is used by the MSs to report back the channel quality measurements from three serving SAs, belonging to three adjacent BSs. A fast cell selection mechanism enables dynamic assignment of the serving SA within a cooperation group. In our system model, simultaneous transmissions from different SAs are separated by CDMA. However, each SA implements TDMA scheme to transmit to different users. Hybrid TDMA/CDMA allows simultaneous transmissions from several SAs to an arbitrary number of users. The system specifications are supported in the new generation of cellular systems, such as cdma2000 $1 \times$ EV-DV [13] and High-Speed Downlink Packet Access (HSDPA) [14].

\section{Cooperative Fair Scheduling Schemes}

In this section, we formulate fair scheduling as an optimization problem, propose the CUFS scheme, develop a flexible fairness enforcement mechanism to improve service smoothness of the CUFS scheme, and investigate the impacts of cooperative scheduling on multiuser diversity gain.

\section{A. Problem Formulation}

Our objective is to maximize the sum of long term throughput of the three SAs. The constraints are fairness and total allowed power budget of the three SAs. Let $m_{j}(t)$ be the index of the serving SA of user $j$ in time slot $t$. Denote by $p_{m_{j}(t), j}(t)$ the allocated transmission power from the $\mathrm{SA}_{m_{j}(t)}$ to user $j$ at time slot $t$. The Signal to Noise and Interference Ratios (SINRs) at the receiver of user $j$ in time slot $t$ are given by

$$
\operatorname{SINR}_{j}(t)=\frac{\left|h_{m_{j}(t), j}(t)\right|^{2} p_{m_{j}(t), j}(t)}{\sum_{l=1, l \neq j}^{N}\left|h_{m_{l}(t), j}(t)\right|^{2} p_{m_{l}(t), i}(t)+\eta_{j}(t)}
$$

where $\left|h_{m_{j}(t), j}(t)\right|^{2}$ is the channel power gain from $\mathrm{SA}_{m_{j}(t)}$ to user $j$ at time slot $t, N$ is the total number of users within a cell (the gray hexagon in Fig. 2), and $\eta_{j}(t)$ is the additive noise power at the receiver of user $j$ in time slot $t$. Assuming that a perfect coding can achieve a transmission rate of Shannon's upper bound, the normalized instantaneous transmission rate of user $j$ is given by

$$
r_{j}(t)=\log _{2}\left[1+\operatorname{SINR}_{j}(t)\right] \quad \mathrm{b} / \mathrm{s} / \mathrm{Hz} .
$$

Thus, the long term average transmission rate of user $j$, denoted by $R_{j}$, is given as follows:

$$
R_{j}=\frac{\sum_{t=1}^{\mathrm{T}} t_{\mathrm{s}} * r_{j}(t)}{T}
$$

where $T$ is the fairness interval, and $t_{\mathrm{s}}$ is the time slot length. Fairness interval is defined as the number of time slots within 


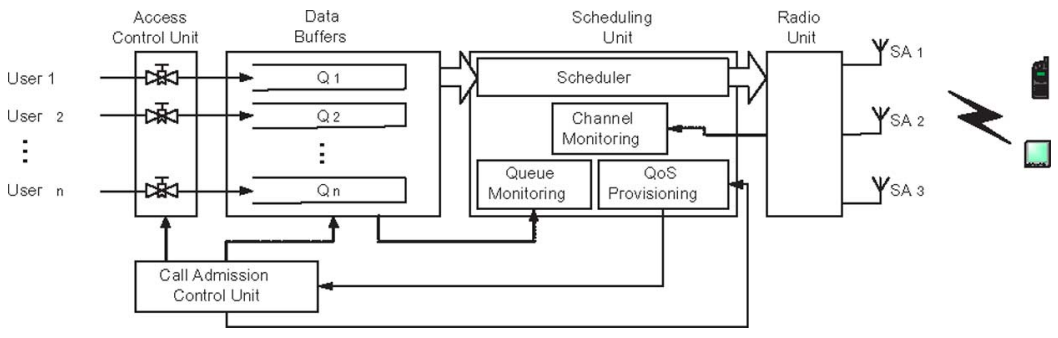

Fig. 3. Resource management module.

that the average transmission rates of all users should satisfy a fairness criterion. The objective of an optimal scheduling scheme is to find the optimum values of $m_{j}(t)$ and $p_{m_{j}(t), j}(t)$ in order to

$$
\begin{aligned}
& \operatorname{maximize} \sum_{j=1}^{N} R_{j} \\
& \text { subject to: } \\
& \sum_{j=1}^{N} p_{m_{j}(t), j}(t)=P, \quad 1 \leq t \leq T \\
& F\left(R_{1}, \ldots, R_{N}\right)=0
\end{aligned}
$$

where $P$ is the maximum transmission power budget. Equation (5) specifies a constraint on the total allowed power budget. $F\left(R_{1}, \ldots, R_{N}\right)=0$ is the fairness constraint which depends on the notion of fairness. We adopt a variant of utility fairness in this paper. Given a rate allocation vector $\left(R_{1}, \ldots, R_{N}\right)$, utility fairness measures the level of fairness by summation of the utility of all users. Utility of a user is defined by a concave utility function. A brief explanation of utility fairness and the motivations for using it as the fairness criterion is given in Appendix A. We also show how to obtain a normalized fair allocation vector which can be used as a reference to measure fairness of the proposed scheduling scheme in Appendix A. The utility fair allocation vector is an allocation vector which specifies the fairest allocation vector among all feasible allocation vectors. It can be used to dynamically monitor the fairness of a scheduling scheme. If the proportions among the actual average allocated rates for different users divert from the fair proportions, the scheduling scheme can adapt itself to improve fairness. Unfortunately, the utility fair allocation vector cannot be obtained if the information about the quality of wireless channels is not available for the entire scheduling period (i.e., $\mathrm{T}$ time slots). In Appendix $\mathrm{A}$, we present an approximation to obtain the utility fair allocation vector for a given scheduling scenario, specified by the average quality of the wireless channels. The proportions are denoted by vector $\left(w_{1}, \ldots, w_{N}\right)$, and referred to as the fair share weights throughout this paper. We consider an allocation vector $\left(R_{1}, \ldots, R_{N}\right)$ as an approximately utility fair allocation vector if

$$
\frac{R_{1}}{w_{1}}=\cdots=\frac{R_{N}}{w_{N}} .
$$

Thus, the optimization problem in (4)-(6) can be simplified as follows:

$$
\operatorname{maximize} \sum_{j=1}^{N} R_{j}
$$

subject to:

$$
\begin{aligned}
& \sum_{j=1}^{N} p_{m_{j}(t), j}(t)=P, \quad 1 \leq t \leq T \\
& \frac{R_{1}}{w_{1}}=\cdots=\frac{R_{N}}{w_{N}} .
\end{aligned}
$$

Solving (8)-(10) to obtain the optimal scheduling is computationally infeasible. However, the formulation of the problem should provide a good guideline which can be used to design efficient heuristic scheduling schemes.

\section{B. Cooperative Scheduling Scheme}

Without a fairness constraint, the optimal scheduling policy is to maximize the instantaneous sum of transmission rates from the three SAs. This problem is an special case of the optimal resource allocation problem for parallel interference channels [16]. To the best of our knowledge, the general solution for this problem has not been obtained yet. However, for a single SA, the problem reduces to an optimal resource allocation for the broadcast Gaussian channels, and the optimal scheduling policy to maximize the total system throughput is to transmit to only one user with the best channel quality in each time slot [6]. We adopt this policy for each of the three SAs in our system model. Each SA transmits to only one user, and three users are selected such that the highest instantaneous transmission rate can be achieved. Unlike independent scheduling for each SA, the data packets of each user may be transmitted from different SAs in different time slots. The transmission power of the SAs is optimized in order to minimize the interference among the SAs. We formulate the optimum power allocation as a nonlinear programming problem in Appendix $\mathrm{B}$. The problem can be solved by a variety of fast numerical methods.

To maintain fairness, we modify the above scheduling strategy by comparing the normalized average throughput of users. Normalization is achieved by dividing the average throughput of user $j$ to its fair share weight $w_{j}$. If the normalized average throughput of users are not equal, the scheduling scheme gives higher priorities to the users with relatively low average 
transmission rates. In other words, the fair scheduling scheme maximizes the sum of instantaneous transmission rates while maintaining the $R_{j} / w_{j}$ ratios approximately equal. The closer the vector $\left(R_{1} / w_{1}, \ldots, R_{N} / w_{N}\right)$ is to an even vector, the higher is the degree of fairness. To achieve this, the scheduler needs to continuously estimate the average transmission rates over the past $T$ time slots. We adopt a low-pass filtering technique from [8] to estimate the average transmission rates. The scheduler updates the estimations at each time slot by

$$
\widetilde{R}_{j}(t)=\left(1-\frac{1}{T_{j}}\right) \widetilde{R}_{j}(t-1)+\frac{1}{T_{j}} r_{j}(t)
$$

where $\widetilde{R}_{j}(t)$ is the estimation of $R_{j}(t)$, and $r_{j}(t)$ is the allocated transmission rate for user $j$ in time slot $t . T_{j}$ is the time constant of the low-pass filter for user $j$. We develop an algorithm to compute $T_{j}$ in the next section.

An efficient fairness mechanism should also consider the deviations among the average quality of the channels for different users. When the deviation is large, a high degree of unfairness is expected; therefore, strong fairness provision is required. The maximum deviation among the average quality of channels is given by

$$
\sigma=\max _{i, j}\left\{a_{i, j}^{(\mathrm{av})}\right\}-\min _{i, j}\left\{a_{i, j}^{(\mathrm{av})}\right\}
$$

where $a_{i, j}^{(\text {av) }}$ is the average quality of the channel for user $j$ from $\mathrm{SA}_{i}$. Let $r_{i, j}(t)$ be the achievable rate from $\mathrm{SA}_{i}$ to user $j$ in time slot $t$. To factor $\sigma$ into the scheduling scheme, we define a normalized achievable rate from $\mathrm{SA}_{i}$ to user $j$ in time slot $t$ as

$$
r_{i, j}^{\prime}(t)=\frac{r_{i, j}(t)}{Y_{j}(t)^{(1+\sigma)}}
$$

where

$$
Y_{j}(t)=\frac{\frac{\widetilde{R}_{j}(t)}{w_{j}}}{\frac{\sum_{j=1}^{N} \frac{\widetilde{R}_{j}(t)}{w_{j}}}{N}} .
$$

$Y_{j}(t)$ represents the ratio between the normalized average transmission rate of user $j$ to the mean normalized average transmission rate of all users. $Y_{j}(t)>1$ means that user $j$ has received less than its fair share in the past $T_{j}$ time slots, and vice versa.

In summary, the CUFS scheme can be implemented in the following steps.

1) MS $j$ estimates its channel power gain $\left|h_{1, j}(t)\right|^{2}$, $\left|h_{2, j}(t)\right|^{2}$, and $\left|h_{3, j}(t)\right|^{2}$ from the three SAs in the associated cooperation group. The estimated values are reported back to a coordination agent through the SA that the MS has been originally associated with. The coordination agent could be the BSC or one of the SAs in a cooperation group. To minimize the signaling load the SAs only exchange the information of the MSs with backlogged data.

2) The coordination agent computes the normalized achievable rates, $r_{i, j}^{\prime}(t)$, by using (13) for $i=1,2,3$ and $j=$ $1, \ldots, N$.

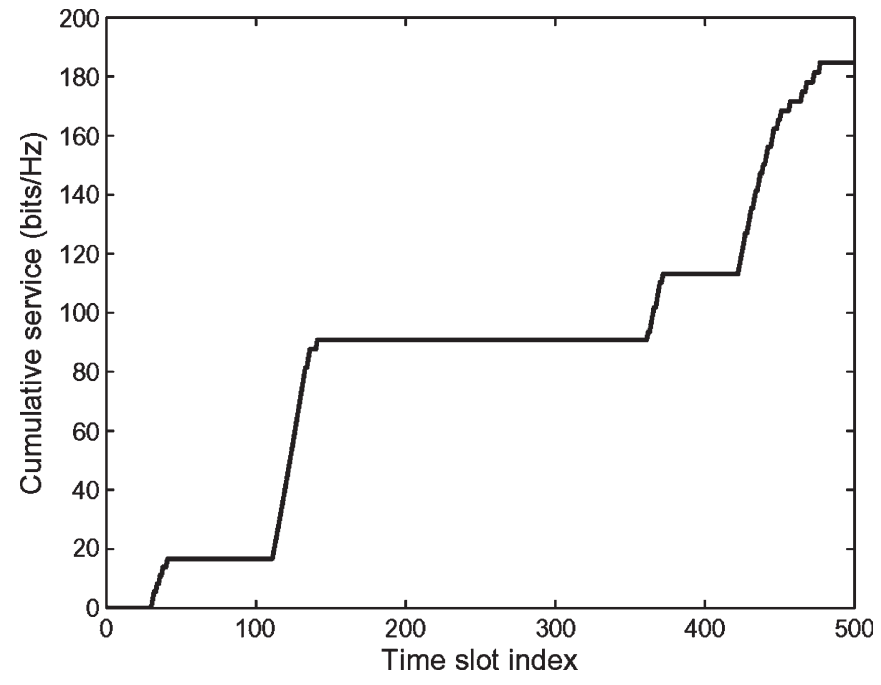

Fig. 4. Nonsmooth service delivery of an opportunistic service discipline.

3) The coordination agent selects three MSs with the highest $r_{i, j}^{\prime}(t)$ for the next time slot. For example, if $r_{2,5}^{\prime}(t)$, $r_{1,15}^{\prime}(t)$, and $r_{3,10}^{\prime}(t)$ are the three highest normalized achievable rates, $\mathrm{SA}_{2}$ transmits to $\mathrm{MS} 5, \mathrm{SA}_{1}$ transmits to MS 15 , and $\mathrm{SA}_{3}$ transmits to MS 10 in the next time slot.

4) For the selected combination of SAs and MSs, the optimal power allocation and achievable rates are computed using the algorithm in Appendix B.

5) The SAs exchange a portion of the backlogged data if necessary.

6) The coordination agent updates $\widetilde{R_{j}}$ for all of the MSs.

\section{Improving Service Smoothness}

In addition to achieving high bandwidth utilization and long term fairness, it is also desirable to provide smooth service for users in order to avoid long periods of starvation. An opportunistic service discipline with the aforementioned long term fairness mechanism can cause a nonsmooth service delivery. Some users may not receive any service for a long period of time and receive abundant service in some other periods of time. This can be illustrated in Fig. 4, where the cumulative service of a user is plotted versus time. To mitigate the problem, we propose an algorithm to compute the value of $T_{j}$ in (11) by using the service smoothness requirement of users $j$. We also define a limit for the minimum value of $\widetilde{R}_{j}(t)$ such that the returning users who have been in idle state for a relatively long period of time do not deprive the other users from transmission for a significant period of time.

Let $R_{j}^{(\mathrm{eff})}$ (b/s) be the effective bandwidth requirement of user $j$, which is the average transmission rate that is required to achieve certain QoS [17]. Denote by $t_{\mathrm{s}}$ and $L_{j}$ the duration of a time slot in seconds and the average packet length of user $j$ in bits, respectively. If a user is served at a transmission rate equal to its effective bandwidth, the average required number of time slots for transmission of a single packet can be given by

$$
M_{j}=\frac{L_{j}}{t_{\mathrm{s}} R_{j}^{(\mathrm{eff})}} .
$$




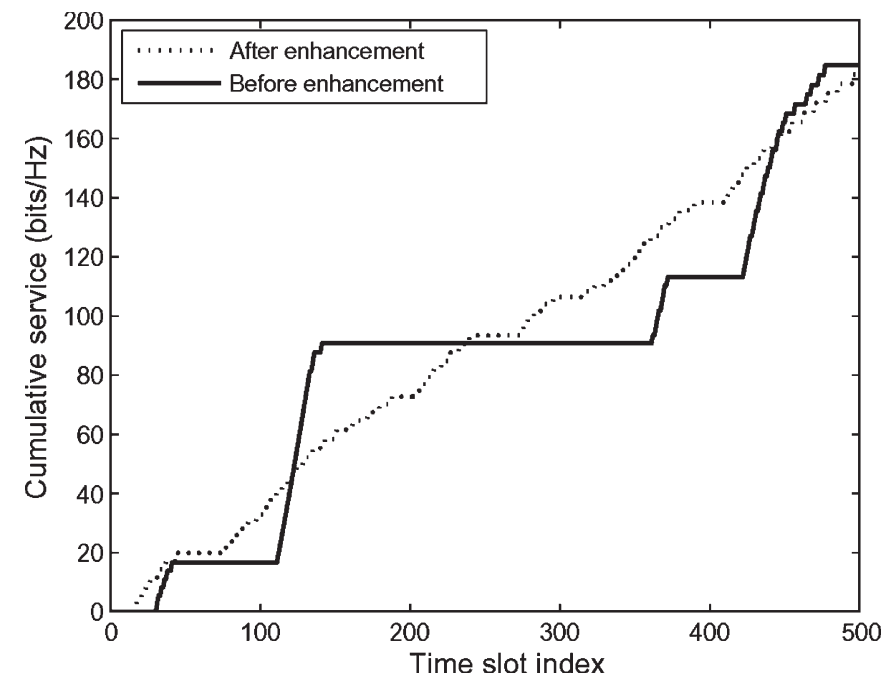

Fig. 5. Enhancement of service smoothness.

We assume that users are admitted with guaranteed effective bandwidth. Denote by $D_{j}$ the average tolerable delay for transmission of a single packet for user $j$. The scheduler should allocate $M_{j}$ time slots to user $j$ in every $D_{j} / t_{\mathrm{s}}$ time slots. This gives an intuitive range for $T_{j}$ in (11) as

$$
T_{j}=\frac{D_{j}}{t_{\mathrm{s}} M_{j}} .
$$

Furthermore, we define a limit for the minimum value of $\widetilde{R}_{j}(t)$ to avoid excessive access to channel by returning users. This can be done by introducing a new update formula for $\widetilde{R}_{j}(t)$ as

$$
Z_{j}(t)=\left(1-\frac{1}{T_{j}}\right) \widetilde{R}_{j}(t-1)+\frac{1}{T_{j}} r_{j}(t)
$$

where

$$
\widetilde{R}_{j}(t)=\max \left\{Z_{j}(t), \beta \cdot R_{j}^{(\mathrm{eff})}\right\}
$$

and $\beta$ is a constant between 0 and 1 . In (17), $Z_{j}(t)$ denotes the estimation of the average transmission rate for user $j$ in the past $T_{j}$ time slots. If $Z_{j}(t)$ is too small such that user $j$ gets a very high chance of transmission for many time slots, its chance is reduced by letting $R_{j}=\beta \cdot R_{j}^{\text {(eff) }}$. Fig. 5 compares the cumulative service of a user before and after the proposed enhancement (for $\beta=0.2$ ). It can be seen that the enhanced scheme provides a significantly smoother service.

\section{Multiuser Diversity Gain of the CUFS Scheme}

We consider a scalar flat fading channel with additive noise. The vector of received symbols at time slot $t$ can be modeled by

$$
\mathbf{y}(t)=\mathbf{h}(t) \mathbf{x}(t)+\mathbf{n}(t)
$$

where $\mathbf{x}(t), \mathbf{h}(t)$, and $\mathbf{n}(t)$ represent the vectors of the transmitted symbols, the channel-gain, and the impact of additive noise plus interference, respectively [16]. We approximate the maximum achievable transmission rate by Shannon's capacity bound for AWGN channels as

$$
r(t)=\log _{2}\left(1+\frac{a(t) p}{\eta}\right) \quad \mathrm{b} / \mathrm{s} / \mathrm{Hz}
$$

where $a(t)=|h(t)|^{2}$ is the channel power gain, $p$ is the transmitter power, and $\eta$ is the spectral power density of the additive noise and interference. For an Ergodic fading process, the Ergodic capacity is defined by

$$
C=E_{a}\left[\log _{2}\left(1+\frac{a p}{\eta}\right)\right]
$$

where $a$ is an exponentially distributed random variable representing the ensemble value of $a(t)$, and $E_{a}$ denotes the expectation of $r(t)$ with respect to $a$ [8].

For a multiuser system, where a single transmitter at a BS is shared among $K$ users, if the transmission is to a single user with the best channel quality (i.e., the highest $a(t)$ at each time slot), the maximum achievable transmission rate at time slot $t$ can be approximated by

$$
r_{K}(t)=\log _{2}\left[1+\frac{p}{\eta} \max _{k}\left(a_{k}(t)\right)\right]
$$

where $k$ is the user index. Extending (21) to the multiuser case, we have

$$
C_{K}=E_{A} \log _{2}\left[1+\frac{p}{\eta} \mathbf{A}\right]
$$

where $\mathbf{A}=\max \left(a_{1}, \ldots, a_{K}\right)$. Consider two distinct scheduling schemes with $K_{1}$ and $K_{2}$ users, respectively. Since $\log _{2}(\cdot)$ is a monotonically increasing function, for any $K_{1} \leq K_{2}$, $C_{K_{1}} \leq C_{K_{2}}$. Thus, the larger the number of users, the larger is the system capacity. The improvement of system capacity due to the number of users is known as multiuser diversity gain.

The aforementioned argument can be used to evaluate multiuser diversity gain of the CUFS scheme. From Fig. 2, let $K_{1}, K_{2}$, and $K_{3}$ be the number of users in the three sectors associated with $\mathrm{SA}_{1}, \mathrm{SA}_{2}$, and $\mathrm{SA}_{3}$, respectively. We compare multiuser diversity gain of a cooperative scheduler with three individual noncooperative schedulers. For the noncooperative schedulers, since a separate scheduler is for each SA, the total Ergodic capacity is $C_{K_{1}}+C_{K_{2}}+C_{K_{3}}$. For the cooperative scheduler, a single scheduler coordinates transmissions from three independent SAs, as specified by the CUFS scheme. Since the cooperative scheduler selects three users for transmission from a pool of $K_{1}+K_{2}+K_{3}$ users, the Ergodic capacity is $C_{K_{1}+K_{2}+K_{3}}+C_{K_{1}+K_{2}+K_{3}}+C_{K_{1}+K_{2}+K_{3}}$. In other words, the cooperative scheduling increases multiuser diversity gain by virtually increasing the effective number of users for each scheduler.

\section{Simulation Results}

We use a standard 19-cell simulation model [18], as shown in Fig. 6. The model accommodates one center cell and 18 interfering cells in two tiers. Each cell is covered by three SAs, 


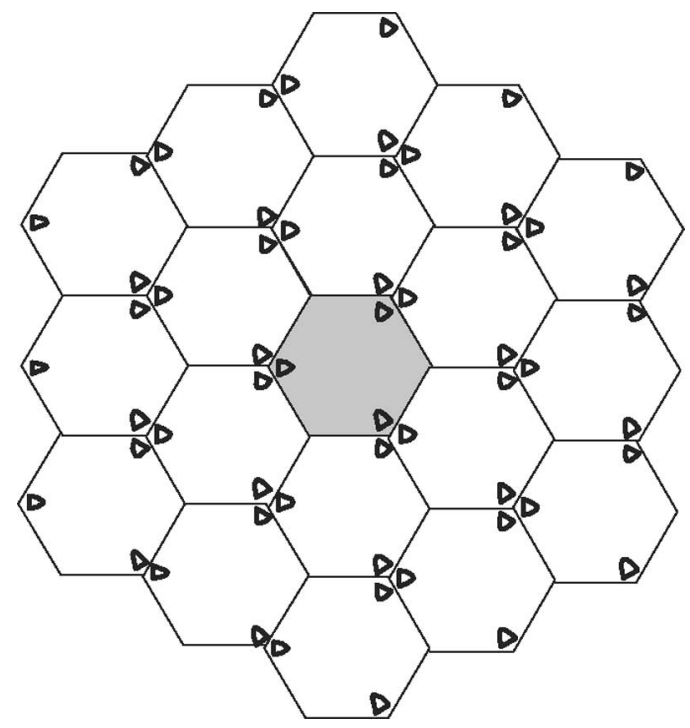

Fig. 6. Simulation model for a cellular network.

TABLE I

Simulation PaRAmeters OF THE FADing ChanNEls

\begin{tabular}{lc}
\hline Parameter & Value \\
\hline Carrier frequency & $1800 \mathrm{Mhz}$ \\
Velocity of mobile nodes & $5 \mathrm{Km} / \mathrm{h}$ \\
Sampling frequency & $1000 \mathrm{samples} \mathrm{per} \mathrm{second}$ \\
Length of a time slot & $1.25 \mathrm{~ms}$ \\
\hline
\end{tabular}

and there are 57 SAs. The maximum power budget of each SA is $1 \mathrm{~W}$; thus, the maximum total power budget of a group of three $\mathrm{SAs}$, covering a single cell, is $3 \mathrm{~W}$. Scheduling is only performed in the center cell. However, interference from all other SAs are considered. Wireless channels from the interfering SAs to the users in the center cell are modeled by considering path loss only. However, channels from the SAs of the center cell to the users in the center cell are modeled by considering path loss, shadowing, and fast Rayleigh fading. Samples of Rayleigh fading are generated by low pass filtering of two white Gaussian random sequences. The parameters of the channel simulator are summarized in Table I. The transmitted signals from different SAs are separated by suborthogonal spreading codes.

We simulate the PFS and the CUFS schemes since both schemes implement a similar opportunistic service discipline. The comparison of the two schemes should demonstrate the impacts of cooperative scheduling. For the PFS scheme, we implement three independent schedulers for three different SAs of the center cell. For the CUFS scheme, a cooperative scheduler coordinates transmission from the three SAs covering the center cell. The normalized values of different parameters for the schedulers are given in Table II. To evaluate the fairness of the schedulers, we use Gini fairness index [19] as follows:

$$
I=\frac{1}{2 N^{2} \bar{u}} \sum_{k=1}^{N} \sum_{l=1}^{N}\left|u_{k}-u_{l}\right|
$$

where $u_{k}=\widetilde{R}_{k} / w_{k}, \mathbf{u}=\left(u_{1}, \ldots, u_{N}\right)$, and $\bar{u}=\left(\sum_{k=1}^{N} u_{k}\right) /$ $N . \widetilde{R}_{k}$ and $w_{k}$ are the long term average transmission rate and the fair share weight of user $k$, respectively. To ensure accuracy of the results, Monte Carlo simulations for one million time
TABLE II

PARAMETERS OF THE CUFS SCHEME

\begin{tabular}{lcl}
\hline Parameter & Value & Comments \\
\hline$R_{j}^{(\min )}$ & 0 & $j=1, \ldots, N$ \\
$R_{j}^{(\max )}$ & $\infty$ & $j=1, \ldots, N$ \\
$T_{j}$ & 500 time slots & $j=1, \ldots, N$ \\
$\beta$ & 0.1 & \\
\hline
\end{tabular}

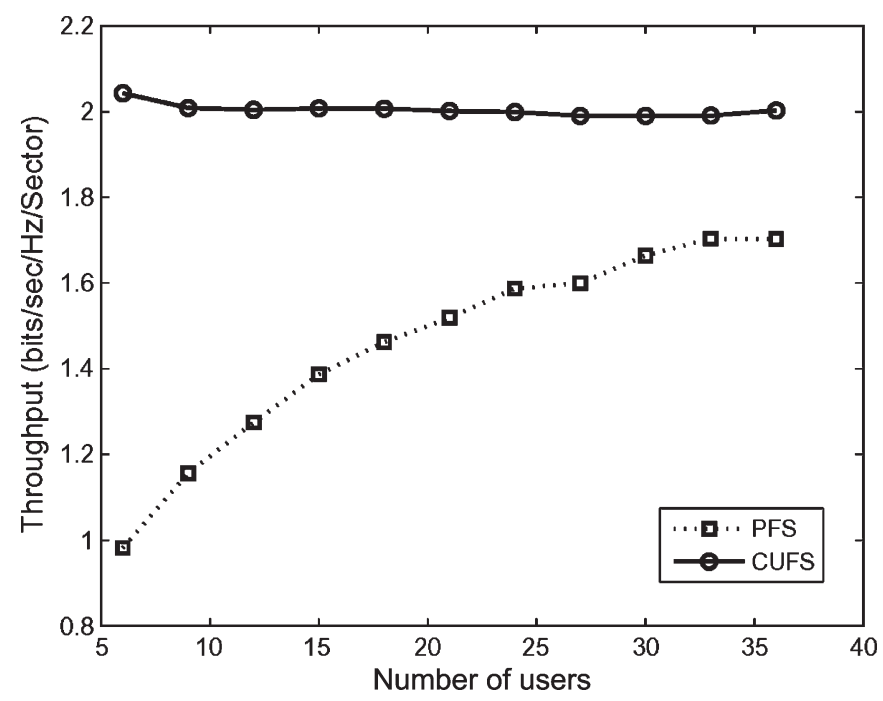

Fig. 7. Throughput for symmetric user distribution.

slots have been performed for each result. We explain the results in the following two sections.

\section{A. Symmetric Users}

By symmetry, the average channel gains from three SAs to a particular user are identical. Although this is rarely a practical scenario, it can be used as a benchmark to evaluate the performance of the scheduling schemes. For each user, the received signal power from three SAs is equal. This means that the interference among the three SAs is strong. The total throughput and fairness index in the center cell versus the number of users are shown in Figs. 7 and 8, respectively. It can be seen that the CUFS scheme significantly outperforms the PFS scheme in terms of throughput and fairness when there is a strong level of interference among SAs. As the number of users increases, the throughput, as well as the level of unfairness of the PFS scheme increase. In contrary, the CUFS scheme sacrifices the inherent multiuser diversity gain to maintain fairness as the number of users increases.

\section{B. Asymmetric Users}

Asymmetry means that each user has different channel gains from the three SAs. The interference among the SAs is weak for a high asymmetric user distribution. We define a normalized parameter, namely symmetry coefficient, to quantify the level of symmetry. When the symmetry coefficient is one, the system is fully symmetric similar to Section V-A. In other words, the interference among the three SAs is the highest. On the other hand, when the symmetry coefficient is zero, the user 


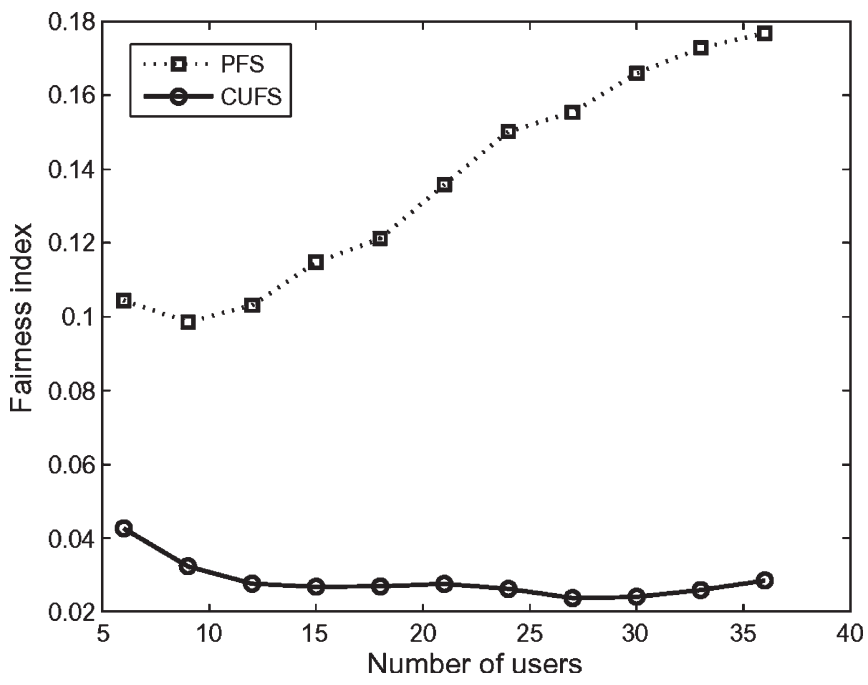

Fig. 8. Fairness index for symmetric user distribution.

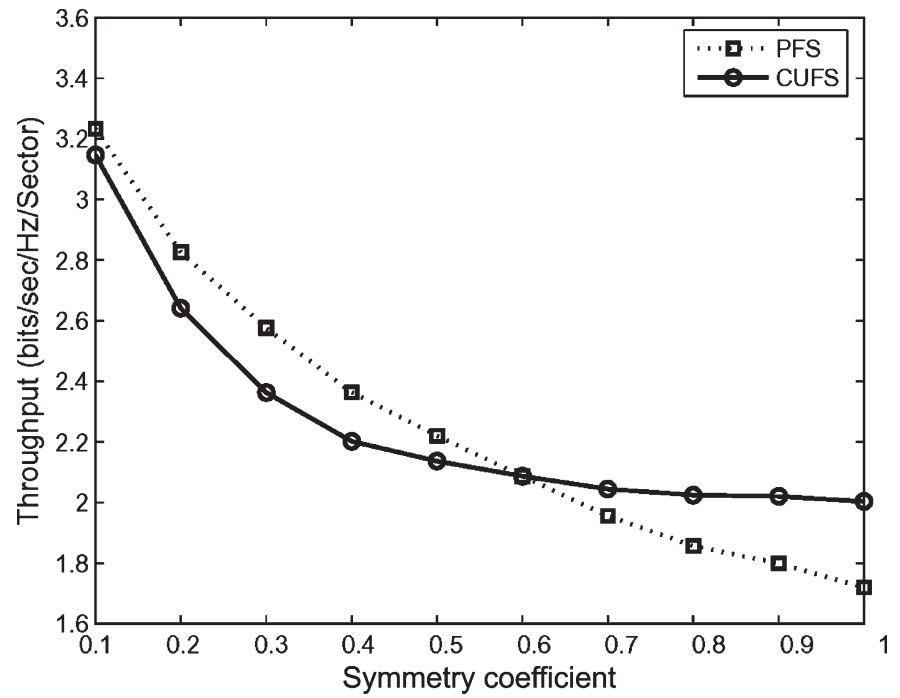

Fig. 9. Throughput versus symmetry coefficient.

distribution is highly asymmetric, and the interference among SAs is the lowest.

For asymmetric user distribution, the fairness index and the throughput versus the symmetry coefficient are shown in Figs. 9 and 10 , respectively. The results are obtained with 36 users in the center cell. It can be seen that the PFS scheme outperforms the CUFS scheme in terms of throughput when the interference among SAs is not severe. However, superiority of the PFS scheme in terms of throughput is achieved by a more unfair rate allocation among the users. If we relax the fairness mechanism of the CUFS scheme a little bit, as it can be seen in Figs. 11 and 12, without losing much fairness, the CUFS can outperform the PFS scheme in terms of throughput for the entire range of the symmetry coefficient.

\section{CONCLUSION}

In this paper, we have proposed a cooperative fair scheduling scheme with an opportunistic service discipline as the core for the downlink of CDMA cellular networks. A fairness

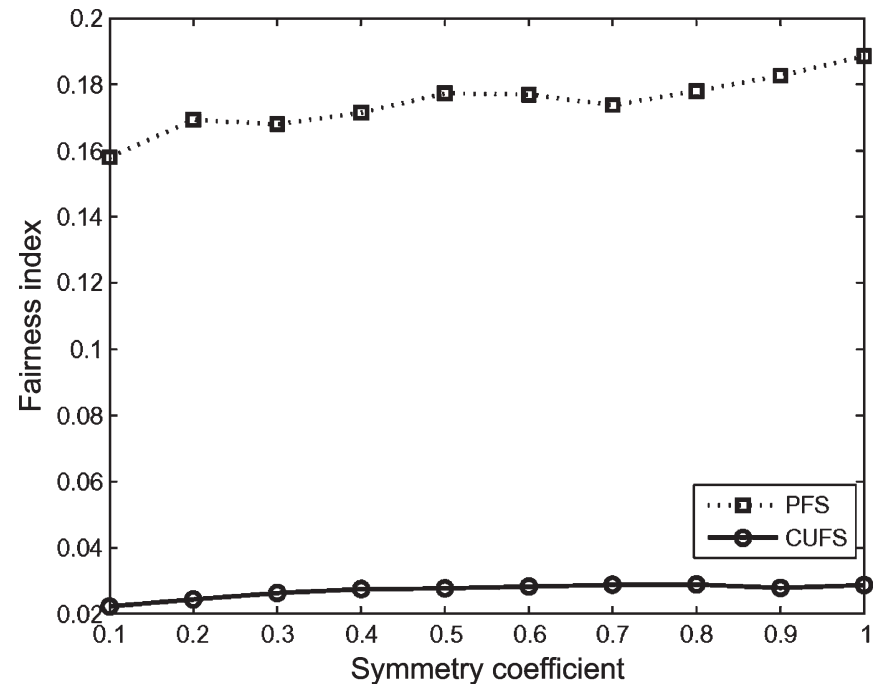

Fig. 10. Fairness index versus symmetry coefficient.

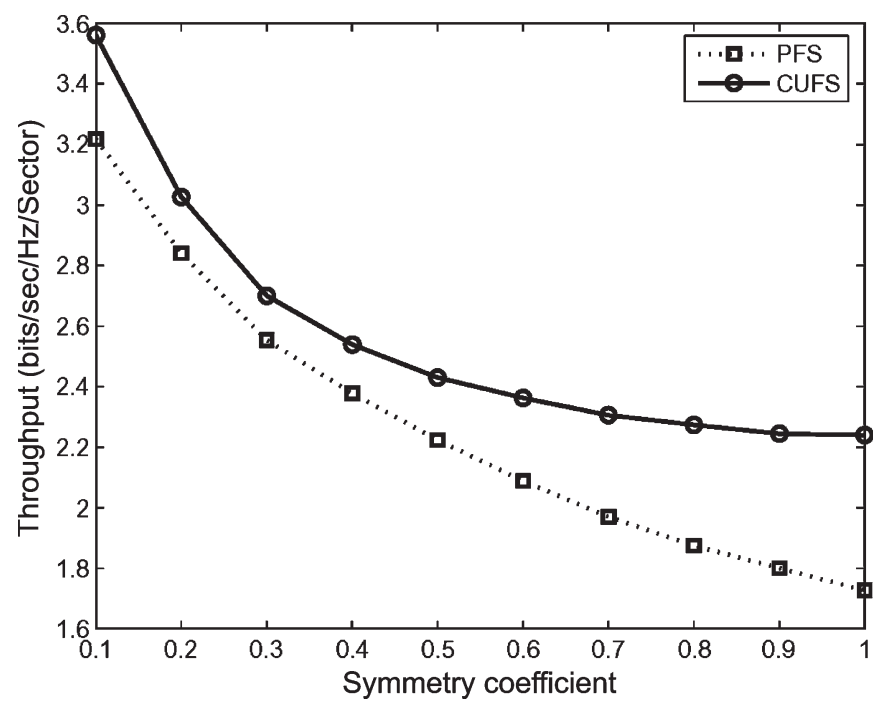

Fig. 11. Throughput versus symmetry coefficient (relaxed fairness mechanism for the CUFS scheme).

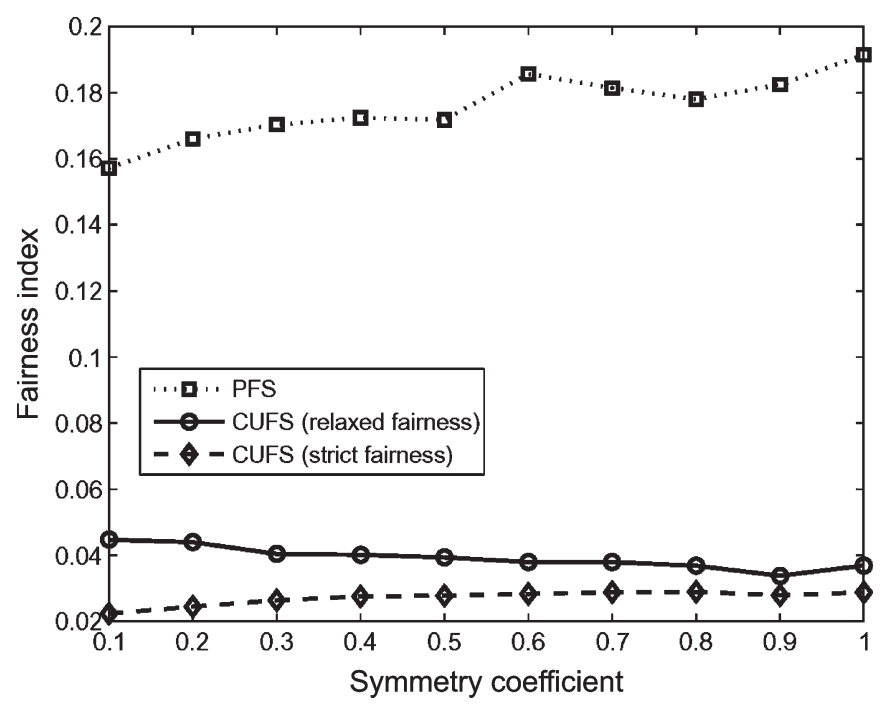

Fig. 12. Fairness index versus symmetry coefficient. 
enforcement mechanism has also been integrated in order to maintain long-term fairness and smooth service delivery. We have shown that a cooperative scheduling increases multiuser diversity gain by increasing the effective number of users. Optimal power allocation among the SAs also reduces interference from the adjacent BSs, thereby improving the total system throughput. Monte Carlo simulation results demonstrate that cooperative scheduling significantly increases system throughput while maintaining a good level of fairness among users. Performance improvement is particularly remarkable for the users located near the cell boundaries where interference among the adjacent BSs is high.

\section{APPENDIX A FAIRNESS}

A proper definition of fairness depends on the application domain. Traditional definitions such as max-min fairness do not properly address the issue in wireless domain [15]. Variations of utility fairness [4] are widely accepted fairness criterion for wireless resource allocation schemes. We adopt a utility-based definition of fairness, i.e., a fair rate allocation vector is the solution of a concave maximization problem as follows:

$$
\left\{\begin{array}{l}
\max _{R} H(\mathbf{R})=\sum_{j=1}^{N} U_{j}\left(R_{j}\right) \\
F(\mathbf{R})=0
\end{array}\right.
$$

where $\mathbf{R}=\left(R_{1}, \ldots, R_{N}\right)$ is the rate allocation vector, $R_{j}$ is the allocated rate to user $j, U_{j}(\cdot)$ is the utility function of user $j, N$ is the number of users, and $F(r)=0$ specifies a set of constraints. The utility function is a concave function. ${ }^{1}$ The relation between the maximization problem in (25) and the concept of fairness can be explained by a simple example as follows. Consider a resource allocation problem with $N$ users. Let all of the $N$ users have similar utility functions, given by $U_{j}(x)=\ln (x+1)$, and the sum of the allocated rates be limited by some value, e.g., $\sum_{j} R_{j}=1$. The fair rate allocation vector can be obtained by solving the following optimization problem:

$$
\left\{\begin{array}{l}
\max _{R} H(\mathbf{R})=\sum_{j=1}^{N} \ln \left(R_{j}+1\right) \\
\sum_{j=1}^{N} R_{j}=1
\end{array} .\right.
$$

The Lagrange function for (26) can be written as

$$
J\left(R_{1}, \ldots, R_{N}\right)=\sum_{j=1}^{N} \ln \left(R_{j}+1\right)+\lambda \sum_{j=1}^{N} R_{j}
$$

where $\lambda$ is the Lagrange multiplier. Differentiating (27) with respect to $R_{j}$, we have

$$
\frac{1}{R_{j}+1}+\lambda=0
$$

\footnotetext{
${ }^{1} \mathrm{~A}$ function $f(x)$ is concave on an interval $[a, b]$ if for any two points $x_{1}$ and $x_{2}$ in $[a, b], f\left[(1 / 2)\left(x_{1}+x_{2}\right)\right] \geq(1 / 2)\left[f\left(x_{1}\right)+f\left(x_{2}\right)\right]$.
}

Applying the constraint $\sum_{j=1}^{N} R_{j}=1$, the fair rate allocation vector can be found as $(1 / N, \ldots, 1 / N)$. The solution is an even rate allocation vector. We can extend the result to any other form of concave utility functions. In other words, if the utility functions are identical and concave, the utility fair allocation vector is an even vector. Fair allocation vector depends on the definition of utility functions. Let $\mathbf{x}=\mathbf{R} \cdot \alpha, x_{\min }=R_{\min }$. $\alpha$, and $x_{\max }=R_{\max } \cdot \alpha$ be the weighted average rate, the weighted minimum average rate, and the weighted maximum average rate of a user, respectively. $\alpha$ is a weighting factor which will be explained later. We define the utility function of a user as

$$
U(x)= \begin{cases}\ln (1+x), & x<x_{\min } \\ \sqrt{\ln (1+x)}+b, & x_{\min } \leq x \leq x_{\max } \\ \sqrt[4]{\ln (1+x)}+c, & x>x_{\max }\end{cases}
$$

where $b$ and $c$ are embedded to force continuity of the utility function, and are given by

$$
\begin{aligned}
b= & \ln \left(1+x_{\min }\right)-\sqrt{\ln \left(1+x_{\min }\right)} \\
c= & \ln \left(1+x_{\max }\right)+\ln \left(1+x_{\min }\right) \\
& -\sqrt{\ln \left(1+x_{\min }\right)}-\sqrt[4]{\ln \left(1+x_{\max }\right) .}
\end{aligned}
$$

The minimum and maximum average rates define a desired operating region for the user to make the utility function sensitive to the allocated rate. Allocating a rate below $R_{\min }$ is considered as a significant performance degradation, and allocating a rate beyond $R_{\max }$ does not increase the actual utility of a user. These parameters differentiate the utility functions of different users. The scaling factor, $\alpha \geq 1$, determines the proximity of the fair allocation vector to an even rate allocation vector. For a large $\alpha$, the solution of (25) is close to an even allocation vector.

A simple two-user power allocation problem can be used to demonstrate the properties of the proposed utility function. Let $\left(x_{\min }, x_{\max }\right)$ be $(3,7)$ and $(2,9)$ for users 1 and 2 , respectively. Using (29), the utility function can be obtained, as shown in Fig. 13. Let the channel power gains be $a_{1}=0 \mathrm{~dB}$ and $a_{2}=$ $10 \mathrm{~dB}$ for users 1 and 2, respectively. The total power budget is constrained by

$$
p_{1}+p_{2}=1
$$

where $p_{1}$ and $p_{2}$ are the transmission power of users 1 and 2, respectively. The fair rate and power allocation vectors can be obtained by solving the following optimization problem:

$$
\left\{\begin{array}{l}
\max _{\left(p_{1}, p_{2}\right)} \quad H\left(x_{1}, x_{2}\right)=U_{1}\left(x_{1}\right)+U_{2}\left(x_{2}\right) \\
x_{j}=\alpha \cdot R_{j} \\
R_{j}=\log _{2}\left(1+a_{j} p_{j}\right) \quad \mathrm{b} / \mathrm{s} / \mathrm{Hz} \\
p_{1}+p_{2}=1
\end{array} .\right.
$$




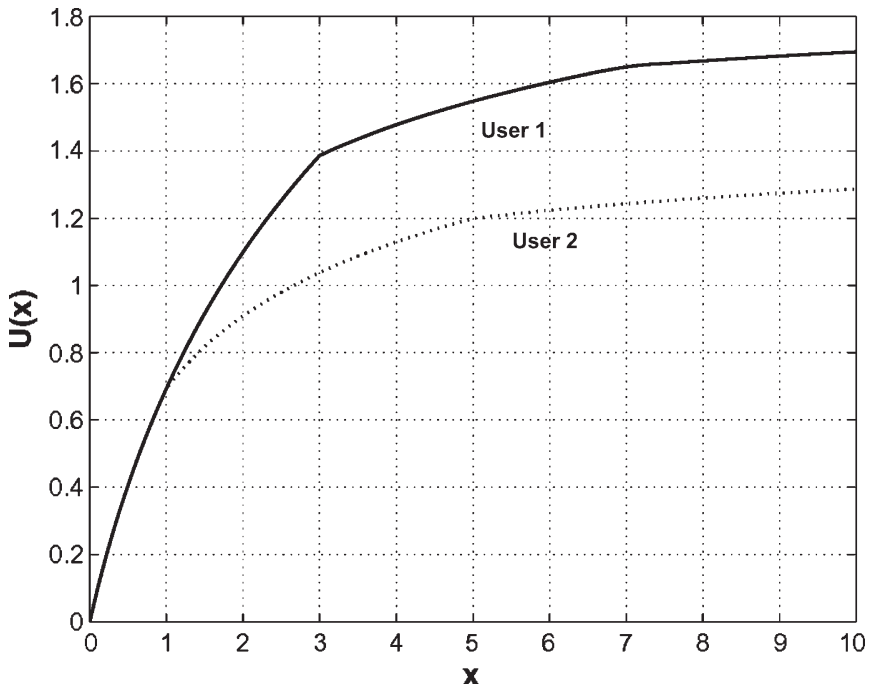

Fig. 13. Two sample utility functions.

For instance, if

$$
\left\{\begin{array}{l}
\text { User } 1: R_{\text {min }}=0.3, R_{\text {max }}=0.7 \\
\text { User } 2: R_{\text {min }}=0.1, R_{\text {max }}=0.5 \\
\alpha=1
\end{array}\right.
$$

the fair power allocation vector is $\left(p_{1}=0.68, p_{2}=0.32\right)$, and the fair rate allocation vector is $\left(R_{1}=0.37, R_{2}=1.03\right)$. If

$$
\left\{\begin{array}{l}
\text { User } 1: R_{\text {min }}=0.3, R_{\text {max }}=0.7 \\
\text { User } 2: R_{\text {min }}=0.1, R_{\text {max }}=0.5 \\
\alpha=1
\end{array}\right.
$$

the fair allocation vector is $\left(p_{1}=0.77, p_{2}=0.23\right)$, and the fair power allocation vector is $\left(R_{1}=0.41, R_{2}=0.85\right)$.

Increasing the value of $\alpha$ results in more even rate distribution but with more unfair power (i.e., effort) allocation and reduction in the total system throughput. In other words, smaller values of $\alpha$ result in more even effort distribution, and larger values of $\alpha$ yield more even rate distributions. To demonstrate the impacts of $x_{\min }$ and $x_{\max }$, let

$$
\left\{\begin{array}{l}
\text { User } 1: R_{\text {min }}=0.6, R_{\max }=0.7 \\
\text { User } 2: R_{\text {min }}=0.1, R_{\text {max }}=0.5 \\
\alpha=10
\end{array} .\right.
$$

The fair power allocation vector is $\left(p_{1}=0.87, p_{2}=0.13\right)$, and the fair rate allocation vector is $\left(R_{1}=0.45, R_{2}=0.59\right)$. It can be seen that as $R_{\min }$ of user 1 is increased from 0.3 in (33) to 0.6 in (33), its allocated power is increased from $p_{1}=0.77$ to $p_{1}=$ 0.87 . Sensitivity of the fair allocation vector to $R_{\max }$ can also be seen, similarly. Thus, the proposed notion of fairness 1 ) is sensitive to QoS; 2) effort unfairness; and 3) service unfairness. Furthermore, $\alpha$ can be used to adjust sensitivity to effort and service unfairness.

We use the aforementioned notion of fairness to define fair share weights, denoted by $\left(w_{1}, \ldots, w_{N}\right)$. The fair share weights

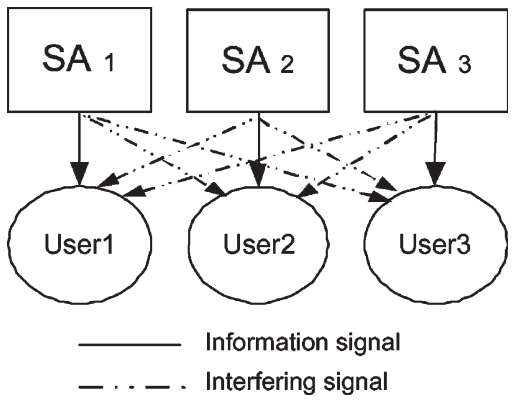

Fig. 14. Power allocation scenario.

are used to evaluate fairness of resource allocation schemes in a similar system settings. Consider the following optimization problem:

$$
\left\{\begin{array}{l}
\max _{\left(p_{1}, \ldots, p_{N}\right)} \quad H\left(x_{1}, \ldots, x_{N}\right)=\sum_{j=1}^{N} U_{j}\left(x_{j}\right) \\
x_{j}=\alpha \cdot R_{j} \\
R_{j}=\log _{2}\left(1+a_{j} p_{j}\right) \quad \mathrm{b} / \mathrm{s} / \mathrm{Hz} \\
\sum_{j=1}^{N} p_{j}=P
\end{array}\right.
$$

where $a_{j}$ is the average channel power gain of user $j, U_{j}(\cdot)$ is the utility function of user $j$, defined by (29), and $P$ is the total power budget. The nonlinear optimization problem in (33) can be solved by nonlinear programming techniques. After computing $p_{j}$ by solving (33), the fair share weights is obtained by

$$
w_{j}=\log _{2}\left(1+a_{j} p_{j}\right) \text {. }
$$

\section{APPENDIX B \\ Optimal Power Allocation}

We develop an optimal power allocation scheme for a scenario illustrated in Fig. 14. In this scenario, $\mathrm{SA}_{i}$ intends to transmit to user $i$; however, the received signal by user $i$ will be affected by the interference of the other two SAs. The objective is to divide a limited power budget among the SAs in order to maximize the sum of transmission rates. Assuming scalar flat fading channels from the SAs to the mobile users, the instantaneous received signals by user 1,2 , and 3 can be represented, respectively, by

$$
\begin{aligned}
& Y_{1}(t)=h_{1,1}(t) X_{1}(t)+h_{2,1}(t) X_{2}(t)+h_{3,1}(t) X_{3}(t)+n_{1}(t) \\
& Y_{2}(t)=h_{1,2}(t) X_{1}(t)+h_{2,2}(t) X_{2}(t)+h_{3,2}(t) X_{3}(t)+n_{2}(t) \\
& Y_{3}(t)=h_{1,3}(t) X_{1}(t)+h_{2,3}(t) X_{2}(t)+h_{3,3}(t) X_{3}(t)+n_{3}(t)
\end{aligned}
$$

where $X_{i}(t)$ is the transmitted signal from $\mathrm{SA}_{i}$ to user $i, Y_{i}(t)$ is the received signal by user $i, h_{i, j}(t)$ is the complex Gaussian channel gain from $\mathrm{SA}_{i}$ to user $j$ at time slot $t$, and $n_{i}(t)$ is the 
additive noise power seen by user $i$. The SINR seen by the users can be given by

$$
\begin{aligned}
\operatorname{SINR}_{1} & =\frac{\left|h_{1,1}\right|^{2} p_{1}}{\left|h_{2,1}\right|^{2} p_{2}+\left|h_{3,1}\right|^{2} p_{3}+\eta_{1}} \\
\operatorname{SINR}_{2} & =\frac{\left|h_{2,2}\right|^{2} p_{1}}{\left|h_{1,2}\right|^{2} p_{1}+\left|h_{3,2}\right|^{2} p_{3}+\eta_{2}} \\
\operatorname{SINR}_{3} & =\frac{\left|h_{3,3}\right|^{2} p_{1}}{\left|h_{1,3}\right|^{2} p_{1}+\left|h_{2,3}\right|^{2} p_{2}+\eta_{2}}
\end{aligned}
$$

where $p_{i}$ is the transmission power of $\mathrm{SA}_{i}$, and $\eta_{i}$ is the power of the additive noise seen by user $i$. To simplify the manipulations, we have dropped time slot index $t$ in (36). Assuming that the fading envelopes are constant for the entire time slot duration and the noise-plus-interference has a Gaussian distribution, Shannon's capacity formula can be used to specify the maximum achievable rates as follows:

$$
\begin{array}{ll}
r_{1}=\log _{2}\left(1+\operatorname{SINR}_{1}\right) & \mathrm{b} / \mathrm{s} / \mathrm{Hz} \\
r_{2}=\log _{2}\left(1+\operatorname{SINR}_{2}\right) & \mathrm{b} / \mathrm{s} / \mathrm{Hz} \\
r_{3}=\log _{2}\left(1+\operatorname{SINR}_{3}\right) & \mathrm{b} / \mathrm{s} / \mathrm{Hz}
\end{array}
$$

where $r_{i}$ is the achievable transmission rate from $\mathrm{SA}_{i}$ to user $i$. Let $P$ denote the total power budget, i.e., $p_{1}+p_{2}+p_{3}=$ $P$. The system capacity region is defined as a region in the 3-D space $\left(r_{1}, r_{r}, r_{3}\right)$, where all rate tuples are achievable by appropriate coding and power allocation schemes. The optimal power allocation problem is to find the power allocation vector $\left(p_{1}, p_{2}, p_{3}\right)$ that maximizes the sum of transmission rates. This can be specified by the following optimization problem:

$$
\left\{\begin{array}{l}
\max _{\left(p_{1}, p_{2}, p_{3}\right)} \quad r_{\mathrm{s}}=r_{1}+r_{2}+r_{3} \\
p_{1}+p_{2}+p_{3}=P
\end{array}\right.
$$

where $r_{\mathrm{s}}$ is the sum of transmission rates. Equations (36)-(38) specify a small scale constrained nonlinear programming problem that can be solved by fast numerical algorithms such as sequential quadratic programming [20].

\section{ACKNOWLEDGMENT}

The authors would like to thank the valuable suggestions from anonymous reviewers.

\section{REFERENCES}

[1] H. Zhang, "Service disciplines for guaranteed performance service in packet-switching networks," Proc. IEEE, vol. 83, no. 10, pp. 1374-1396, Feb. 1995.

[2] S. Lu and V. Bharghavan, "Fair scheduling in wireless packet networks," IEEE/ACM Trans. Netw., vol. 7, no. 4, pp. 473-489, Aug. 1999.

[3] S. N. Diggavi, N. Al-Dhahir, A. Stamouls, and A. R. Calderbank, "Great expectations: The value of spatial diversity in wireless networks," Proc. IEEE, vol. 92, no. 2, pp. 219-270, Feb. 2004.

[4] A. Maullloo, F. P. Kelly, and D. Tan, "Rate control in communication networks: Shadow prices, proportional fairness and stability," J. Oper. Res. Soc., vol. 49, no. 3, pp. 237-252, Mar. 1998.
[5] P. Bhagwat, A. Krishna, and S. Tripathi, "Enhancing throughput over wireless LANs using channel state dependent packet scheduling," in Proc. INFOCOM, Mar. 1996, pp. 1133-1140.

[6] R. Knopp and P. A. Humblet, "Information capacity and power control in single cell multiuser communications," in Proc. IEEE Int. Conf. Commun., Jun. 1995, pp. 331-335.

[7] D. N. C. Tse, "Optimal power allocation over parallel Gaussian channels," in Proc. Int. Symp. Inf. Theory, Jun. 1997, p. 27.

[8] A. Jalali, R. Padovalni, and R. Pankaj, "Data throughput of CDMA-HDR a high efficiency data rate," in Proc. IEEE VTC-Spring, 2000, vol. 1.3, pp. $1854-1858$

[9] P. Viswanath, D. N. C. Tse, and R. Laria, "Opportunistic beamforming using dumb antennas," IEEE Trans. Inf. Theory, vol. 48, no. 6, pp. $1277-$ 1294, Jun. 2002.

[10] S. Borst and P. Whiting, The use of diversity antennas in high-speed wireless systems: Capacity gain, fairness, multi-user scheduling, Bell Lab., Lucent Technol. [Online]. Available: http://mars.bell-labs.com/cm/ $\mathrm{ms} /$ what/mars/papers

[11] D. Aktas and H. El Gamal, "Multiuser scheduling for multiple antenna systems," in Proc. IEEE Pac. Rim Conf. Comput. Process., Aug. 2003, vol. 1, pp. 502-505.

[12] D. J. Mazzarese and W. A. Krzymien, "High throughput downlink cellular packet data access with multiple antennas and multiuser diversity," in Proc. IEEE VTC, Apr. 2003, vol. 2, pp. 1079-1083.

[13] 3GPP2, High speed data enhancements for cdma2000 1X-Integrated data and voice, Oct. 2000. S.R0026, Version 1.0.

[14] 3GPP, UTRA high speed downlink packet access, Mar. 2001. TR 25.950 V4.0.0.

[15] M. Dianati, X. Shen, and S. Naik, "A new fairness index for radio resource allocation in wireless networks," in Proc. IEEE WCNC, Mar. 13-17, 2005, vol. 2, pp. 712-717.

[16] T. M. Cover and J. A. Thomas, Elements of Information Theory. New York: Wiley, 1991.

[17] G. Kesidis, J. Walrand, and C. S. Chang, "Effective bandwidths for multiclass Markov fluids and other ATM sources," IEEE/ACM Trans. Netw., vol. 1, no. 4, pp. 424-428, Aug. 1993.

[18] 3GPP2 WG5 Evaluation Ad Hoc, 1xEV-DV Evaluation Methodology Addendum (V6), Jul. 2001.

[19] A. W. Marshall and I. Olkin, Inequalites: Theory of Majorization and Its Applications. New York: Academic, 1979.

[20] K. Schittkowski, "NLQPL: A FORTRAN-subroutine solving constrained nonlinear programming problems," Ann. Oper. Res., vol. 5, no. 1-4, pp. $485-500,1985$.

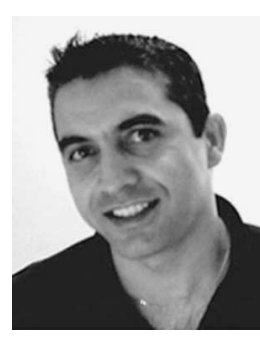

Mehrdad Dianati (S'06) received the B.S. and M.S. degrees in electrical engineering from Sharif University of Technology, Tehran, Iran, in 1992 and K.N.Toosi University of Technology, Tehran, in 1995, respectively, and the Ph.D. degree in electrical and computer engineering from University of Waterloo, Waterloo, ON, Canada, in 2006.

From 1995 to 1997, he was a Lecturer with Aerospace University, Tehran. He was a Hardware and Software Developer from 1997 to 2002 . He is currently a Postdoctoral Research Associate at Ecole Nationale Superieure des Telecommunication, Paris, France. 


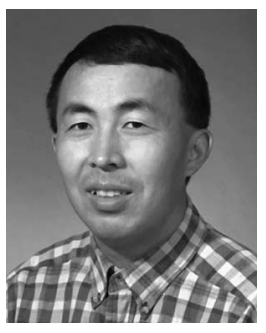

Xuemin (Sherman) Shen (M'97-SM'02) received the B.Sc. degree from Dalian Maritime University, Dalian, China, in 1982 and the M.Sc. and Ph.D. degrees from Rutgers University, Camden, NJ, in 1987 and 1990, respectively, all in electrical engineering.

From September 1990 to September 1993, he was first with the Howard University, Washington, DC, and then with the University of Alberta, Edmonton, AB, Canada. Since October 1993, he has been with the Department of Electrical and Computer Engineering, University of Waterloo, Waterloo, ON, Canada, where he is a Professor and the Associate Chair for Graduate Studies. His research focuses on mobility and resource management in interconnected wireless/wireline networks, ultrawideband wireless communications systems, wireless security, and ad hoc and sensor networks. He is a coauthor of two books and has published more than 200 papers and book chapters on wireless communications and networks, control, and filtering.

Dr. Shen served as the Technical Program Committee Chair for Qshine'05, Cochair for IEEE Broadnet'05, WirelessCom'05, IFIP Networking'05, ISPAN'04, and the IEEE Globecom'03 Symposium on Next Generation Networks and Internet. He also serves as the Associate Editor for the IEEE TRANSACTIONS ON WIRELESS COMMUNICATIONS; IEEE TRANSACTIONS ON VehICUlaR TEChNOlogy; ACM Wireless Network; Computer Networks; Dynamics of Continuous, Discrete and Impulsive-Series B: Applications and Algorithms; Wireless Communications and Mobile Computing (Wiley); International Journal Computer and Applications; and he was a Guest Editor for the IEEE Journal on SELECTEd AREAs in COMMUnications, IEEE Wireless Communications, and IEEE Communications Magazine. He received the Premier's Research Excellence Award from the Province of Ontario, Canada, for demonstrated excellence of scientific and academic contributions in 2003 and the Distinguished Performance Award from the Faculty of Engineering, University of Waterloo, for outstanding contributions in teaching, scholarship, and service in 2002. He is a registered Professional Engineer in Ontario.

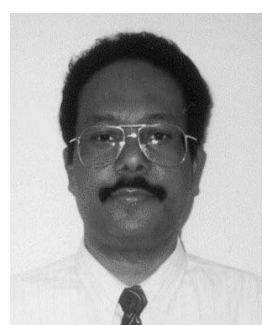

Kshirasagar Naik (M'94) received the B.S. degree from Sambalpur University, Orissa, India, the M.Tech. degree from Indian Institute of Technology, Kharagpur, India, the M. Math degree in computer science from University of Waterloo, Waterloo, $\mathrm{ON}$, Canada, and the Ph.D. degree in electrical and computer engineering from Concordia University, Montreal, QC, Canada.

He was a Faculty Member at the University of Aizu, Japan, and Carleton University, Ottawa, ON. Currently, he is an Associate Professor with the Department of Electrical and Computer Engineering, University of Waterloo. He was a Visiting Associate Professor at the Research Institute of Electrical Communications, Tohoku University, Sandai, Japan, during May-November 2003. His research interests include testing of communication protocols, wireless communication, resource allocation in cellular networks sensor networks, ad hoc networks, MAC protocols, personal area networks, mobile computing, and peer-to-peer communication.

Dr. Naik served as a Program Cochair of the 5th International Conference on Information Technology held in Bhubaneswar, India, in December 2002. He was a Co-Guest Editor of a special issue of the IEEE JOURNAL ON SELECTED AREAS IN COMMUNICATIONS (JSAC) on mobile computing and networking published in June 2005. Currently, he is a Co-Guest Editor of a special issue of IEEE JSAC on peer-to-peer communications and applications. 